\title{
Natural quasi-alignment with two Higgs doublets and RGE stability
}

\author{
F. J. Botella ${ }^{1, \mathrm{a}}$, G. C. Branco ${ }^{2,3,4, \mathrm{~b}}$, António M. Coutinho ${ }^{2, \mathrm{c}}$, M. N. Rebelo ${ }^{2, \mathrm{~d}}$, J. I. Silva-Marcos ${ }^{2, \mathrm{e}}$ \\ ${ }^{1}$ Departament de Física Teòrica and IFIC, Universitat de València-CSIC, 46100 Burjassot, Spain \\ ${ }^{2}$ Centro de Física Teórica de Partículas (CFTP), Instituto Superior Técnico (IST), Universidade de Lisboa (UL), Avenida Rovisco Pais, $1049-001$ \\ Lisbon, Portugal \\ ${ }^{3}$ Departamento de Física, Instituto Superior Técnico (IST), Universidade de Lisboa (UL), Avenida Rovisco Pais, 1049-001 Lisbon, Portugal \\ 4 Theory Group, Physics Department, CERN, 1211 Geneva 23, Switzerland
}

Received: 23 February 2015 / Accepted: 27 May 2015 / Published online: 26 June 2015

(C) The Author(s) 2015. This article is published with open access at Springerlink.com

\begin{abstract}
In the context of two Higgs doublet models, we study the conditions required in order to have stable quasialignment in flavour space. We show that stability under the renormalisation group equations imposes strong constraints on the flavour structure of the Yukawa couplings associated to each one of the Higgs doublets. In particular, we find a novel solution, where all Yukawa couplings are proportional to the so-called democratic matrix. This solution is rather unique, since it is the only stable solution which is a good starting point for reproducing the observed pattern of quark masses and mixing. We also show that this stable solution can be obtained by imposing on the Lagrangian a $Z_{3} \times Z_{3}^{\prime}$ flavour symmetry. Quark masses of the lighter quark generations are generated through the breaking of this discrete symmetry, and, at this stage, scalar-mediated flavour-changing neutralcurrents arise, but they are naturally suppressed by the smallness of the light quark masses. In this way, we relate Higgs alignment to the hierarchy of the quark masses through a discrete family symmetry.
\end{abstract}

\section{Introduction}

One of the simplest extensions of the standard model (SM) consists of the addition of scalar doublets to the SM spectrum. Multi-Higgs extensions arise in a variety of frame-

\footnotetext{
a e-mail: fbotella@uv.es

b e-mail: gbranco@tecnico.ulisboa.pt

c e-mail: antonio.coutinho@tecnico.ulisboa.pt

de-mail: rebelo@tecnico.ulisboa.pt

e e-mail: juca@cftp.ist.utl.pt
}

works, including supersymmetric extensions of the SM, as well as models with family symmetries. A two Higgs doublet model (2HDM) was first introduced by Lee [1], in order to achieve spontaneous breaking of the CP symmetry. If no extra symmetries are introduced, 2HDMs lead to too large tree-level scalar-mediated flavour-changing neutral-currents (FCNCs) [2,3]. In order to avoid these potentially dangerous currents, various schemes have been proposed:

(i) Glashow and Weinberg [4] have pointed out that one can avoid FCNCs at tree level by introducing a $Z_{2}$ symmetry under which the two Higgs doublets transform differently. The introduction of a $Z_{2}$ symmetry in $2 \mathrm{HDMs}$ prevents the generation of spontaneous $\mathrm{CP}$ breaking [5] unless the symmetry is softly broken [6].

(ii) Pich and Tuzon [7] have conjectured the existence of flavour alignment of the two Yukawa matrices, thus avoiding FCNCs at tree level. This is an interesting suggestion, but it has the drawback of being an ad-hoc assumption, not explained by any symmetry. Furthermore, it has been pointed out that in general this scheme is not stable under the renormalisation group [8]. There have been attempts to obtain alignment in various extensions of the SM [9-11].

(iii) Another possibility has been proposed some time ago [12] by Branco, Grimus and Lavoura (BGL) who have pointed out that there is a symmetry which, when imposed on the Lagrangian, constrains the Yukawa couplings in such a way that FCNCs do arise at tree level, but are entirely determined by the $V_{\mathrm{CKM}}$ matrix, with no other free parameters. In some of the BGL models, one has a strong natural suppression of the most dangerous FCNC, with, for example, the strangenesschanging neutral currents, proportional to $\left(V_{t d} V_{t s}^{*}\right)^{2}$, 
which implies a very strong natural suppression of the contribution to the $K^{0}-\bar{K}^{0}$ transition. With this suppression, the neutral Higgs masses need not be too large. BGL models have been extended to the leptonic sector [13], their relation to minimal flavour violation models has been studied [14] and their phenomenological implications have been recently analysed [15-17].

This is not a complete list of possible schemes to avoid large FCNC in 2HDM, some other plausible solutions consist in postulating concrete Yukawa structures, such as the renowned Cheng and Sher [18] ansatz, or in simply decoupling the heavy Higgs states.

In this paper, we reexamine the question of the stability of flavour alignment under the renormalisation group. Assuming that the Yukawa couplings of the two Higgs doublets are aligned, i.e., proportional to each other, we study under what conditions the alignment is maintained by the renormalisation group. Apart from the conditions already found in Ref. [8], we find new solutions which can be of great physical interest. One of these solutions, corresponds to having all the Yukawa coupling matrices proportional to the so-called democratic matrix [19-21]. This solution is rather unique, since on the one hand it is stable under the renormalisation group equations (RGEs) and on the other hand, it is the only stable solution which provides a good starting point for reproducing the observed pattern of quark masses and mixing. We then point out that this flavour democratic solution can be obtained as a result of a $Z_{3} \times Z_{3}^{\prime}$ flavour symmetry. In the framework that we propose, flavour alignment is exact in the limit where only the third family acquires mass. Once the two light generations acquire a mass, there are small deviations from alignment, which are suppressed by the strong hierarchy of quark masses. As a result, one obtains in this framework, a quasi-alignment of the Yukawa couplings, as a result of the $Z_{3} \times Z_{3}^{\prime}$ symmetry, together with the strong hierarchy of quark masses.

The paper is organised as follows. In the next section, we briefly describe the general flavour structure of the 2HDM, in order to settle our notation. In Sect. 3 we derive all the solutions for the Yukawa couplings, leading to alignment, stable under the renormalisation group. In Sect. 4 we show that the flavour democratic solution can be obtained as a result of a $Z_{3} \times Z_{3}^{\prime}$ flavour symmetry and propose an ansatz for the breaking of the $Z_{3} \times Z_{3}^{\prime}$ symmetry. In Sect. 5, we examine the suppression of scalar-mediated FCNCs in our framework. In Sect. 6, we perform a numerical analysis, showing how the pattern of quark masses and mixing can be obtained in the framework of our ansatz. Finally our conclusions are contained in Sect. 7. In the appendix we present a full study of the solutions of the alignment conditions.

\section{Yukawa couplings in the general two-Higgs-doublet-model (2HDM)}

For completeness and in order to establish our notation we briefly review the flavour structure of the $2 \mathrm{HDM}$, when no extra symmetries are introduced in the Lagrangian. The Yukawa couplings can be written:

$$
\begin{aligned}
\mathcal{L}_{\mathrm{Y}}= & -\overline{Q_{L}^{0}} \Gamma_{1} \Phi_{1} d_{R}^{0}-\overline{Q_{L}^{0}} \Gamma_{2} \Phi_{2} d_{R}^{0}-\overline{Q_{L}^{0}} \Omega_{1} \tilde{\Phi}_{1} u_{R}^{0} \\
& -\overline{Q_{L}^{0}} \Omega_{2} \tilde{\Phi}_{2} u_{R}^{0}-\overline{L_{L}^{0}} \Pi_{1} \Phi_{1} l_{R}^{0}-\overline{L_{L}^{0}} \Pi_{2} \Phi_{2} l_{R}^{0}+\text { h.c. }
\end{aligned}
$$

where $\Phi_{i}$ denote the Higgs doublets and $\widetilde{\Phi}_{i} \equiv i \tau_{2} \Phi_{i}^{*}$, and $\Gamma_{i}, \Omega_{i}$ and $\Pi_{i}$ are matrices in flavour space. After spontaneous symmetry breaking, the following quark mass matrices are generated:

$M_{d}=\frac{1}{\sqrt{2}}\left(v_{1} \Gamma_{1}+v_{2} e^{i \alpha} \Gamma_{2}\right)$,
$M_{u}=\frac{1}{\sqrt{2}}\left(v_{1} \Omega_{1}+v_{2} e^{-i \alpha} \Omega_{2}\right)$

where $v_{i} / \sqrt{2} \equiv|<0| \phi_{i}^{0}|0>|$ and $\alpha$ denotes the relative phase of the two vacuum expectation values (vevs) of the neutral components $\phi_{i}^{0}$ of $\Phi_{i}$. The neutral and the charged Higgs interactions with quarks are of the form

$$
\begin{aligned}
\mathcal{L}_{\mathrm{Y}}(\text { quark, Higgs })= & -\overline{d_{L}^{0}} \frac{1}{v}\left[M_{d} H^{0}+N_{d}^{0} R+i N_{d}^{0} I\right] d_{R}^{0} \\
& -\overline{u_{L}^{0}} \frac{1}{v}\left[M_{u} H^{0}+N_{u}^{0} R+i N_{u}^{0} I\right] u_{R}^{0} \\
& +\frac{\sqrt{2} H^{+}}{v}\left(\overline{u_{L}^{0}} N_{d}^{0} d_{R}^{0}-\overline{u_{R}^{0}} N_{u}^{0^{\dagger}} d_{L}^{0}\right)+\text { h.c. }
\end{aligned}
$$

where $v \equiv \sqrt{v_{1}^{2}+v_{2}^{2}} \approx 246 \mathrm{GeV}$, and $H^{0}$ and $R$ are orthogonal combinations of the fields $\rho_{j}$, arising when one expands [1] the neutral scalar fields around their vevs, $\phi_{j}^{0}=$ $\frac{e^{i \alpha_{j}}}{\sqrt{2}}\left(v_{j}+\rho_{j}+i \eta_{j}\right)$. Here we should choose $H^{0}$ in such a way that it has couplings to the quarks which are proportional to the mass matrices, as can be seen from Eq. (3). Similarly, I denotes the linear combination of $\eta_{j}$ orthogonal to the neutral Goldstone boson. The matrices $N_{d}^{0}$ and $N_{u}^{0}$ are given by

$N_{d}^{0}=\frac{1}{\sqrt{2}}\left(v_{2} \Gamma_{1}-v_{1} e^{i \alpha} \Gamma_{2}\right)$

$N_{u}^{0}=\frac{1}{\sqrt{2}}\left(v_{2} \Omega_{1}-v_{1} e^{-i \alpha} \Omega_{2}\right)$.

The quark mass matrices are diagonalised through

$U_{d L}^{\dagger} M_{d} U_{d R}=D_{d} \equiv \operatorname{diag}\left(m_{d}, m_{s}, m_{b}\right)$,

$U_{u L}^{\dagger} M_{u} U_{u R}=D_{u} \equiv \operatorname{diag}\left(m_{u}, m_{c}, m_{t}\right)$,

and the matrices $N_{d}^{0}$ and $N_{u}^{0}$ in the mass eigenstate basis transform into

$U_{d L}^{\dagger} N_{d}^{0} U_{d R}=N_{d}, \quad U_{u L}^{\dagger} N_{u}^{0} U_{u R}=N_{u}$. 
There are similar expressions for the leptonic sector. We do not introduce neutrino masses since these are not relevant for our analysis.

\section{Stability of the aligned 2HDM under RGE}

The aligned two Higgs doublet model (A2HDM) is defined at tree level by the following relations involving the matrices introduced in Eq. (1):

$$
\begin{aligned}
& \Gamma_{2}=d \cdot \Gamma_{1} \\
& \Omega_{2}=u \cdot \Omega_{1} \\
& \Pi_{2}=e \cdot \Pi_{1}
\end{aligned}
$$

where $d, u$ and $e$ are constants. In this section we analyse the stability of the A2HDM under the RGEs. The one loop RGEs for the Yukawa couplings are $[8,22]$

$$
\begin{aligned}
\mathcal{D} & \Gamma_{k}=a_{\Gamma} \Gamma_{k} \\
& +\sum_{l=1}^{2}\left[3 \operatorname{Tr}\left(\Gamma_{k} \Gamma_{l}^{\dagger}+\Omega_{k}^{\dagger} \Omega_{l}\right)+\operatorname{Tr}\left(\Pi_{k} \Pi_{l}^{\dagger}+\Sigma_{k}^{\dagger} \Sigma_{l}\right)\right] \Gamma_{l} \\
& +\sum_{l=1}^{2}\left(-2 \Omega_{l} \Omega_{k}^{\dagger} \Gamma_{l}+\Gamma_{k} \Gamma_{l}^{\dagger} \Gamma_{l}+\frac{1}{2} \Omega_{l} \Omega_{l}^{\dagger} \Gamma_{k}+\frac{1}{2} \Gamma_{l} \Gamma_{l}^{\dagger} \Gamma_{k}\right) \\
\mathcal{D} & \Omega_{k}=a_{\Omega} \Omega_{k} \\
& +\sum_{l=1}^{2}\left[3 \operatorname{Tr}\left(\Omega_{k} \Omega_{l}^{\dagger}+\Gamma_{k}^{\dagger} \Gamma_{l}\right)+\operatorname{Tr}\left(\Sigma_{k} \Sigma_{l}^{\dagger}+\Pi_{k}^{\dagger} \Pi_{l}\right)\right] \Omega_{l} \\
& +\sum_{l=1}^{2}\left(-2 \Gamma_{l} \Gamma_{k}^{\dagger} \Omega_{l}+\Omega_{k} \Omega_{l}^{\dagger} \Omega_{l}+\frac{1}{2} \Gamma_{l} \Gamma_{l}^{\dagger} \Omega_{k}+\frac{1}{2} \Omega_{l} \Omega_{l}^{\dagger} \Omega_{k}\right) \\
\mathcal{D} & \Pi_{k}=a_{\Pi} \Pi_{k} \\
& +\sum_{l=1}^{2}\left[3 \operatorname{Tr}\left(\Gamma_{k} \Gamma_{l}^{\dagger}+\Omega_{k}^{\dagger} \Omega_{l}\right)+\operatorname{Tr}\left(\Pi_{k} \Pi_{l}^{\dagger}+\Sigma_{k}^{\dagger} \Sigma_{l}\right)\right] \Pi_{l} \\
& +\sum_{l=1}^{2}\left(-2 \Sigma_{l} \Sigma_{k}^{\dagger} \Pi_{l}+\Pi_{k} \Pi_{l}^{\dagger} \Pi_{l}+\frac{1}{2} \Sigma_{l} \Sigma_{l}^{\dagger} \Pi_{k}+\frac{1}{2} \Pi_{l} \Pi_{l}^{\dagger} \Pi_{k}\right),
\end{aligned}
$$

where $\mathcal{D} \equiv 16 \pi^{2} \mu(\mathrm{d} / \mathrm{d} \mu)$ and $\mu$ is the renormalisation scale. The coefficients $a_{\Gamma}, a_{\Omega}$ and $a_{\Pi}$ are given by

$$
\begin{aligned}
& a_{\Gamma}=-8 g_{s}^{2}-\frac{9}{4} g^{2}-\frac{5}{12} g^{\prime 2}, \\
& a_{\Omega}=-8 g_{s}^{2}-\frac{9}{4} g^{2}-\frac{17}{12} g^{\prime 2}, \\
& a_{\Pi}=-\frac{9}{4} g^{2}-\frac{15}{4} g^{\prime 2}
\end{aligned}
$$

where $g_{s}, g$ and $g^{\prime}$ are the gauge coupling constants of $\mathrm{SU}(3)_{c}, \mathrm{SU}(2)_{L}$ and $U(1)_{Y}$, respectively. The alignment relations given by Eq. (7) guarantee the absence of Higgs- mediated FCNCs at tree level because both matrices $M_{d}$ and $N_{d}$ are proportional to $\Gamma_{1}$. Similarly both $M_{u}$ and $N_{u}$ are proportional to $\Omega_{1}$ and $M_{l}, N_{l}$ to $\Pi_{1}$. In general, these relations are broken at one loop level. From Eqs. (8)-(10) one can easily derive

$$
\begin{aligned}
& \mathcal{D}\left(\Gamma_{2}\right)-d \cdot \mathcal{D}\left(\Gamma_{1}\right) \\
& =\left(u^{*}-d\right)(1+u d)\left\{3 \operatorname{Tr}\left(\Omega_{1}^{\dagger} \Omega_{1}\right)-2 \Omega_{1} \Omega_{1}^{\dagger}\right\} \Gamma_{1} \\
& \quad+(e-d)\left(1+e^{*} d\right) \operatorname{Tr}\left(\Pi_{1}^{\dagger} \Pi_{1}\right) \Gamma_{1}, \\
& \mathcal{D}\left(\Omega_{2}\right)-u \cdot \mathcal{D}\left(\Omega_{1}\right) \\
& =\left(d^{*}-u\right)(1+u d)\left\{3 \operatorname{Tr}\left(\Gamma_{1}^{\dagger} \Gamma_{1}\right)-2 \Gamma_{1} \Gamma_{1}^{\dagger}\right\} \Omega_{1} \\
& \quad+\left(e^{*}-u\right)(1+e u) \operatorname{Tr}\left(\Pi_{1}^{\dagger} \Pi_{1}\right) \Omega_{1}, \\
& \mathcal{D}\left(\Pi_{2}\right)-e \cdot \mathcal{D}\left(\Pi_{1}\right) \\
& =3(d-e)\left(1+d^{*} e\right) \operatorname{Tr}\left(\Gamma_{1}^{\dagger} \Gamma_{1}\right) \Pi_{1} \\
& \quad+3\left(u^{*}-e\right)(1+e u) \operatorname{Tr}\left(\Omega_{1}^{\dagger} \Omega_{1}\right) \Pi_{1} .
\end{aligned}
$$

In order to enforce Eq. (7) at one loop level it is easy to realise that it is sufficient to impose

$$
\begin{aligned}
& \mathcal{D}\left(\Gamma_{2}\right)-d \cdot \mathcal{D}\left(\Gamma_{1}\right) \propto \Gamma_{1}, \\
& \mathcal{D}\left(\Omega_{2}\right)-u \cdot \mathcal{D}\left(\Omega_{1}\right) \propto \Omega_{1}, \\
& \mathcal{D}\left(\Pi_{2}\right)-e \cdot \mathcal{D}\left(\Pi_{1}\right) \propto \Pi_{1} ;
\end{aligned}
$$

in fact the proportionality constants on the r.h.s. are the running ${ }^{1}$ of $d, u$ and $e$. Therefore, Eq. (16) does not impose any constraint: at one loop level the charged lepton sector remains aligned and there are no FCNCs in the leptonic sector. This result agrees with the findings of Refs. [23,24].

In Eqs. (14) and (15) the pieces that can break the alignment in the quark sector are the terms: $\Omega_{1} \Omega_{1}^{\dagger} \Gamma_{1}$ and $\Gamma_{1} \Gamma_{1}^{\dagger} \Omega_{1}$ respectively. ${ }^{2}$ In order to have alignment at one loop level fulfilling Eqs. (17) and (18) - there are two types of solutions:

1. $\left(u^{*}-d\right)(1+u d)=0$

2. $\Omega_{1} \Omega_{1}^{\dagger} \Gamma_{1}=\lambda_{\Gamma} \Gamma_{1}$ and $\Gamma_{1} \Gamma_{1}^{\dagger} \Omega_{1}=\lambda_{\Omega} \Omega_{1}$. With $\lambda_{\Gamma}$ and $\lambda_{\Omega}$ complex numbers.

Solutions of type 1 include the usual 2HDM with natural flavour conservation, where the up and down quarks receive contributions from only one Higgs doublet. It is well known that this can be achieved through the introduction of a $Z_{2}$

\footnotetext{
1 The authors of Ref. [8] impose the condition that the r.h.s. of Eqs. (14)-(16) be equal to zero. This amounts to imposing alignment at one loop level and imposing additionally that there is no running of the parameters $u, d$ and $e$.

${ }^{2}$ It can be readily seen that $\Omega_{1} \Omega_{1}^{\dagger} \Gamma_{1} \propto M_{u} M_{u}^{\dagger} M_{d}$ and $\Gamma_{1} \Gamma_{1}^{\dagger} \Omega_{1} \propto$ $M_{d} M_{d}^{\dagger} M_{u}$. It is worth emphasizing that these structures are precisely the ones obtained in [23,24], which produce FCNCs at one loop level and break the alignment. Note that this radiatively induced misalignment is in general very small as also argued in $[25,26]$.
} 
symmetry. Here, we are not interested in this class of well known solutions. We are interested in the class of solutions of type 2 , and in the appendix we study the complete set of matrices $\Omega_{1}$ and $\Gamma_{1}$ that obey to the conditions required for this class of solutions. We show in the appendix that if one requires stability under the RGE and at the same time Yukawa structures which are, in leading order, in agreement with the observed pattern of quark masses and mixing, then one is lead to a unique solution, where the matrices $\Omega_{1}$ and $\Gamma_{1}$ are of the form

$\Omega_{1}=c_{1}^{d} \Delta ; \quad \Gamma_{1}=c_{1}^{u} \Delta$

with $\Delta$ the democratic mass matrix:

$\Delta=\left(\begin{array}{lll}1 & 1 & 1 \\ 1 & 1 & 1 \\ 1 & 1 & 1\end{array}\right)$.

This solution corresponds to the limit where only the top and bottom quarks acquire mass, while the two first generations are massless. The up and down quarks are aligned in flavour space, so the $V_{\mathrm{CKM}}$ matrix equals the identity. The other stable solutions of type 2 correspond to non-realistic cases like for example having all up or down quarks massless or two up or two down quark masses degenerate or with a $V_{\mathrm{CKM}}$ very far from the identity matrix.

It is remarkable that the so-called democratic mass matrix is stable under RGE and that precisely this stability also enforces what could be called "quark alignment" in the sense that we also have a proportionality among $\Gamma_{i}$ and $\Omega_{i}$.

\section{Natural quasi-alignment of Yukawa couplings}

In this section we search for the minimal symmetry which when imposed on the Lagrangian, leads to the stable solution described in the previous section, corresponding to the democratic Yukawa couplings of Eq. (20). Before describing this symmetry, it is worth to analyse another type of alignment which is verified experimentally, the so-called up-down alignment in the quark sector.

\subsection{The up-down alignment in the quark sector}

In the quark sector, flavour mixing is small. This means that there is a weak basis (WB) where both $M_{u}$ and $M_{d}$ are close to the diagonal form. Experiment indicates that not only flavour mixing is small, but there is also up-down flavour alignment in the quark sector in the following sense. We can choose, without loss of generality, a WB where $M_{u}=\operatorname{diag}\left(m_{u}, m_{c}, m_{t}\right)$. Of course, this is just a choice of ordering, with no physical meaning. Small mixing implies that in this WB $M_{d}$ is almost diagonal. In principle, since the Yukawa couplings $Y_{u}$ and $Y_{d}$ are not constrained in the
SM, there is equal probability of $M_{d}$ being close to $M_{d}=$ $\operatorname{diag}\left(m_{d}, m_{s}, m_{b}\right)$. corresponding to up-down alignment, or being close, for instance, to $M_{d}=\operatorname{diag}\left(m_{b}, m_{s}, m_{d}\right)$ in which case there is up-down misalignment. It is clear that in the SM, assuming small mixing and hierarchical quark masses, the probability of obtaining up-down alignment is only $1 / 6$. Given a set of arbitrary quark mass matrices $M_{u}$ and $M_{d}$, one can derive necessary and sufficient conditions to obtain small mixing and up-down alignment, expressed in terms of WB invariants [27]. Since the experimentally verified up-down alignment is not automatic in the SM, one may wonder whether there is a symmetry which leads to up-down alignment. In the next subsection, we propose a symmetry which leads to up-down alignment in the quark sector and when extended to a $2 \mathrm{HDM}$ leads to a natural alignment of the two Higgs doublets in flavour space.

\section{2 $Z_{3} \times Z_{3}^{\prime}$ symmetry and the two Higgs alignment}

We introduce the $Z_{3} \times Z_{3}^{\prime}$ symmetry under which the quark left-handed doublets $Q_{L_{i}}^{0}$, the right-handed up quarks $u_{L_{i}}^{0}$ and the right-handed down quarks $d_{L_{i}}^{0}$ transform in the following way:

$$
\begin{gathered}
Q_{L_{i}}^{0} \longrightarrow P_{i j}^{\dagger} Q_{L_{j}}^{0}, \\
u_{R_{i}}^{0} \longrightarrow P_{i j} u_{R_{j}}^{0}, \\
d_{R_{i}}^{0} \longrightarrow P_{i j} d_{R_{j}}^{0},
\end{gathered}
$$

where $Z_{3}$ corresponds to $P=\mathbb{1}+E_{1}$ and $Z_{3}^{\prime}$ to $P=\mathbb{1}+E_{2}$ with

$$
\begin{aligned}
& E_{1}=\frac{\omega-1}{2}\left(\begin{array}{ccc}
1 & -1 & 0 \\
-1 & 1 & 0 \\
0 & 0 & 0
\end{array}\right) \\
& E_{2}=\frac{\omega-1}{3}\left(\begin{array}{ccc}
\frac{1}{2} & \frac{1}{2} & -1 \\
\frac{1}{2} & \frac{1}{2} & -1 \\
-1 & -1 & 2
\end{array}\right)
\end{aligned}
$$

and $\omega=e^{\frac{2 \pi i}{3}}$. The Higgs doublets transform trivially under $Z_{3} \times Z_{3}^{\prime}$. The above symmetry leads to the following form for the Yukawa matrices: $\Gamma_{j}=c_{j}^{d} \Delta, \Omega_{j}=c_{j}^{u} \Delta$, corresponding to the stable solution of Eq. (20). This can easily be checked since $\Delta E_{1}=\Delta E_{2}=0$. We thus conclude that the symmetry of Eqs. (22) and (23) leads to the alignment of the two Yukawa coupling matrices, with a democratic flavour structure. Note that this solution also guarantees an up-down alignment in the quark sector, as defined in the previous subsection.

In order to give mass to the first two quark generations, the $Z_{3} \times Z_{3}^{\prime}$ symmetry has to be broken. This breaking will also lead to Higgs-mediated FCNCs, but these couplings will be suppressed by the smallness of the quark masses. In order to illustrate how a realistic pattern of quark masses and mixing 
can be obtained, we shall assume that the breaking of the $Z_{3} \times Z_{3}^{\prime}$ symmetry occurs in two steps. In the first step the symmetry $Z_{3} \times Z_{3}^{\prime}$ is broken into just one of the $Z_{3}$ and the second generation acquires mass and finally in the last step the masses of the quarks $u$ and $d$ are generated. In the first step the symmetry $Z_{3} \times Z_{3}^{\prime}$ is broken to $Z_{3}$ generated by $P=1+E_{1}$. One can check that

$$
\begin{aligned}
\Gamma_{j} & =c_{j}^{d}\left(\Delta+\varepsilon_{d} A\right) ; \quad \Omega_{j}=c_{j}^{u}\left(\Delta+\varepsilon_{u} A\right) ; \\
A & =\left(\begin{array}{lll}
0 & 0 & 1 \\
0 & 0 & 1 \\
1 & 1 & 1
\end{array}\right)
\end{aligned}
$$

are invariant under this $Z_{3}$ symmetry. Note that $A E_{1}=0$. At this stage the second generation acquires mass. Finally, the lightest quarks, $u$ and $d$ acquire mass through a small perturbation, proportional to $\hat{\delta}_{d, u}$, which breaks this $Z_{3}$ symmetry. We assume that

$\Gamma_{2}=c_{2}^{d}\left(\Delta+\varepsilon_{d} A+\hat{\delta}_{d} B_{d}\right)$,

while

$\Gamma_{1}=c_{1}^{d}\left(\Delta+\varepsilon_{d} A\right)$

with equivalent expressions for the up sector. Here

$B_{u}=\left(\begin{array}{ccc}0 & 0 & 1 \\ 0 & 0 & 0 \\ 1 & 0 & 1\end{array}\right) ; \quad B_{d}=\left(\begin{array}{ccc}0 & 0 & 1 \\ 0 & 0 & 0 \\ 1 & 0 & \eta\end{array}\right)$

where $\eta$ is some complex number with modulus of order one. The symmetry is broken, and neither $B_{u}$ nor $B_{d}$ are invariant under the $Z_{3} \times Z_{3}^{\prime}$ symmetry.

\section{Suppression of scalar-mediated FCNC}

In order to study the suppression of a scalar-mediated FCNC, it is useful to start by analysing the parameter space in our framework.

\subsection{The parameter space}

From Eqs. (2), (25) and (26) it follows that, in leading order, $m_{b}=\frac{3}{\sqrt{2}}\left|c_{1}^{d} v_{1}+c_{2}^{d} v_{2} e^{i \alpha}\right| ; \quad m_{t}=\frac{3}{\sqrt{2}}\left|c_{1}^{u} v_{1}+c_{2}^{u} v_{2} e^{-i \alpha}\right|$.

Writing $v \equiv \sqrt{v_{1}^{2}+v_{2}^{2}}=v_{1} \sqrt{1+t^{2}}$, with

$$
t=\frac{v_{2}}{v_{1}}
$$

we obtain in leading order the following relations:

$$
\frac{\left|c_{1}^{d}+c_{2}^{d} t e^{i \alpha}\right|}{\sqrt{1+t^{2}}}=\frac{\sqrt{2}}{3} \frac{m_{b}}{v} ; \quad \frac{\left|c_{1}^{u}+c_{2}^{u} t e^{-i \alpha}\right|}{\sqrt{1+t^{2}}}=\frac{\sqrt{2}}{3} \frac{m_{t}}{v},
$$

which impose restrictions on the allowed parameter space. A priori, we do not assume any conspiracy between parameters and take $t=O(1)$. It is then clear from Eq. (30) that the $c_{i}^{u}$ are generically of order one, while $c_{i}^{d}$ are smaller and may assume values of order $O\left(\frac{m_{b}}{m_{t}}\right)$. This is an important ingredient which, as we shall see, will play a rôle in the evaluation of the strengths of the FCNCs and the allowed parameter space for the Higgs masses.

Next we give the structure of the flavour-changing neutral Yukawa couplings. To this aim, it is useful to express the quark mass matrices in Eq. (2) in terms of the perturbations given in Eqs. (25) and (26):

$$
\begin{aligned}
& M_{d}=\frac{v_{1}}{\sqrt{2}}\left(c_{1}^{d}+c_{2}^{d} t e^{i \alpha}\right)\left[\Delta+\varepsilon_{d} A+\delta_{d} B_{d}\right] \\
& M_{u}=\frac{v_{1}}{\sqrt{2}}\left(c_{1}^{u}+c_{2}^{u} t e^{-i \alpha}\right)\left[\Delta+\varepsilon_{u} A+\delta_{u} B_{u}\right] ;
\end{aligned} \quad \begin{aligned}
& \delta_{d} \equiv \frac{c_{2}^{d} t e^{i \alpha}}{c_{1}^{d}+c_{2}^{d} t e^{i \alpha}} \widehat{\delta}_{d}, \\
& \delta_{u} \equiv \frac{c_{2}^{u} t e^{-i \alpha}}{c_{1}^{u}+c_{2}^{u} t e^{-i \alpha}} \widehat{\delta}_{u} .
\end{aligned}
$$

Then we derive the expressions for the matrices which couple to the Higgs scalars in Eqs. (3) and (4). In the basis where the up and down quark matrices are diagonal, the matrices $N_{d}$ and $N_{u}$ of Eq. (6) become

$$
\begin{aligned}
& N_{d}=t D_{d}-\frac{v_{1}}{\sqrt{2}}\left(1+t^{2}\right) e^{i \alpha} U_{d_{L}}^{\dagger} \Gamma_{2} U_{d_{R}}, \\
& N_{u}=t D_{u}-\frac{v_{1}}{\sqrt{2}}\left(1+t^{2}\right) e^{-i \alpha} U_{u_{L}}^{\dagger} \Omega_{2} U_{u_{R}}
\end{aligned}
$$

where we have used Eqs. (4) and (6) with Eq. (2). Finally, from Eq. (32) combined with Eqs. (25) and (31) we find

$$
\begin{aligned}
& N_{d}=\frac{c_{1}^{d} t-c_{2}^{d} e^{i \alpha}}{c_{1}^{d}+c_{2}^{d} t e^{i \alpha}} D_{d}-\frac{v}{\sqrt{2}} \frac{c_{1}^{d} \sqrt{1+t^{2}}}{t} \delta_{d} U_{d_{L}}^{\dagger} B_{d} U_{d_{R}} \\
& N_{u}=\frac{c_{1}^{u} t-c_{2}^{u} e^{-i \alpha}}{c_{1}^{u}+c_{2}^{u} t e^{-i \alpha}} D_{u}-\frac{v}{\sqrt{2}} \frac{c_{1}^{u} \sqrt{1+t^{2}}}{t} \delta_{u} U_{u_{L}}^{\dagger} B_{u} U_{u_{R}}
\end{aligned}
$$

where $D_{d} \equiv \operatorname{diag}\left(m_{d}, m_{s}, m_{b}\right)$ and $D_{u} \equiv \operatorname{diag}\left(m_{u}, m_{c}, m_{t}\right)$.

The crucial point is that in our scheme these matrices have an extra suppression factor, proportional to $\delta_{d, u}$. Using the expressions given in Eqs. (31) and (27), and computing the trace, the second invariant and the determinant for the squared quark mass matrices $H_{u, d} \equiv\left(M M^{\dagger}\right)_{u, d}$, one can find that in leading order

$$
\begin{aligned}
& \delta_{d}=\sqrt{3} \sqrt{\frac{m_{d}}{m_{s}}} \frac{m_{s}}{m_{b}}=O\left(\lambda^{3}\right) \\
& \delta_{u}=\sqrt{3} \sqrt{\frac{m_{u}}{m_{c}}} \frac{m_{c}}{m_{t}}=O\left(\lambda^{5}\right)
\end{aligned}
$$

where $\lambda \equiv 0.2$ is of the order of the Cabibbo angle. 
From Eq. (31) it follows that in leading order $U_{d_{L}}=$ $U_{u_{L}}=U_{d_{R}}=U_{u_{R}}=F$, where

$$
F==\left(\begin{array}{ccc}
1 / \sqrt{2} & 1 / \sqrt{6} & 1 / \sqrt{3} \\
-1 / \sqrt{2} & 1 / \sqrt{6} & 1 / \sqrt{3} \\
0 & -2 / \sqrt{6} & 1 / \sqrt{3}
\end{array}\right)
$$

is the matrix that diagonalises the exact democratic limit $\Delta$. Thus, taking into account Eq. (27), the matrix contributions from $U_{d_{L}}^{\dagger} B_{d} U_{d_{R}}$ and $U_{u_{L}}^{\dagger} B_{u} U_{u_{R}}$ are both of order one. One can thus conclude that:

- for the down sector, with the assumptions made after Eq. (30), we have a total suppression factor of $O\left(\frac{m_{b}}{m_{t}}\right) \cdot O\left(\lambda^{3}\right)$

- for the up sector, we have a suppression factor of $O\left(\lambda^{5}\right)$ or smaller depending on the value that we choose to assume for $c_{1}^{u}$, but which, as explained, it is reasonable to take of order one.

\section{Numerical analysis}

The matrices of Eq. (31) may be explicitly written

$$
\begin{gathered}
M_{u}=c_{u}\left(\begin{array}{ccc}
1 & 1 & 1+\varepsilon+\delta \\
1 & 1 & 1+\varepsilon \\
1+\varepsilon+\delta & 1+\varepsilon & 1+\varepsilon+\delta
\end{array}\right)_{u}, \\
M_{d}=c_{d}\left(\begin{array}{ccc}
1 & 1 & 1+\varepsilon+\delta \\
1 & 1 & 1+\varepsilon \\
1+\varepsilon+\delta & 1+\varepsilon & 1+\varepsilon+\eta \delta
\end{array}\right)_{d},
\end{gathered}
$$

where we have introduced $c_{d} \equiv \frac{v_{1}}{\sqrt{2}}\left(c_{1}^{d}+c_{2}^{d} t e^{i \alpha}\right)$ and $c_{u} \equiv \frac{v_{1}}{\sqrt{2}}\left(c_{1}^{u}+c_{2}^{u} t e^{-i \alpha}\right)$. Although these two coefficients are in general complex, and since the physically meaningful matrices are those defined as $H=M M^{\dagger}$, both coefficients may be taken as real for our numerical exercise. If one then parametrises the remaining variables as

$\varepsilon=\varepsilon_{m} \exp \left(i \varepsilon_{f}\right), \quad \delta=\delta_{m} \exp \left(i \delta_{f}\right)$,

$\eta=\eta_{m} \exp \left(i \eta_{f}\right)$,

one is left with 12 real parameters that compose the quark mass matrices in our scheme.

In order to check if this parameter space could accommodate the flavour sector, a numerical survey was made where we looked for one combination that could fit the observed values of the quark masses given at the scale of the $\mathrm{Z}$ boson mass [28], the moduli of the entries of the CKM matrix [29], the strength of $\mathrm{CP}$ violation $I_{\mathrm{CP}}$ and $\sin 2 \beta$ and $\gamma$ [29], with $\beta$ and $\gamma$ being two of the angles of the unitarity triangle. A simple run of all 12 parameters produced a "reference point":

\begin{tabular}{lll}
\hline & Up sector & Down sector \\
\hline$c$ & 56.73 & 0.89 \\
$\varepsilon_{m}$ & $1.6 \times 10^{-2}$ & 0.11 \\
$\varepsilon_{f}$ & $-5.6 \times 10^{-3}$ & 0.41 \\
$\delta_{m}$ & $8.1 \times 10^{-4}$ & $2.2 \times 10^{-2}$ \\
$\delta_{f}$ & $\pi+0.32$ & 2.26 \\
$\eta_{m}$ & - & 4.99 \\
$\eta_{f}$ & - & $\pi+0.62$ \\
\hline
\end{tabular}

which yields the output values:

$$
\begin{aligned}
D_{d} & =\operatorname{diag}(0.00204,0.05824,2.85356) \mathrm{GeV}, \\
D_{u} & =\operatorname{diag}(0.00114,0.61736,171.684) \mathrm{GeV}, \\
\left|V_{\mathrm{CKM}}\right| & =\left(\begin{array}{lll}
0.9745 & 0.2244 & 0.0036 \\
0.2243 & 0.9737 & 0.0415 \\
0.0087 & 0.0407 & 0.9991
\end{array}\right), \\
\left|I_{\mathrm{CP}}\right| & =3.0 \times 10^{-5} \\
\sin 2 \beta & =0.69 \\
\gamma & =69.3^{\circ} .
\end{aligned}
$$

It should be noted that the 12 parameters fix not only $V_{\mathrm{CKM}}$ and the quark mass spectrum, but also the strengths of all the FCNC couplings. In order to evaluate the numerical stability of this reference point, we performed a numerical check, varying the input parameters randomly around the values that produced the reference point above; the new results were then combined in the scatter plots shown in Fig. 1 where the reference point is highlighted. In order to obtain an estimate of the lower bound for the flavour-violating Higgs masses, we consider the contribution to $K^{0}-\bar{K}^{0}$ mixing. Apart from the SM box diagram one now has a new physics contribution arising from the scalar-mediated FCNC tree-level diagrams thus making the total transition amplitude equal to $M_{12}=$ $M_{12}^{\mathrm{SM}}+M_{12}^{\mathrm{NP}}$, Ref. [16] with

$$
\begin{aligned}
M_{12}^{\mathrm{NP}}= & \sum_{H=R, I} \frac{f_{M}^{2} m_{M}}{96 v^{2} m_{H}^{2}}\left\{\left[1+\left(\frac{m_{M}}{m_{q 1}+m_{q 2}}\right)^{2}\right]\right. \\
& \left.\times C_{1}(H)-\left[1+11\left(\frac{m_{M}}{m_{q 1}+m_{q 2}}\right)^{2}\right] C_{2}(H)\right\},
\end{aligned}
$$

where

$C_{1}(R)=\left[\left(N_{q 2 q 1}\right)^{*}+N_{q 1 q 2}\right]^{2}$,
$C_{2}(R)=\left[\left(N_{q 2 q 1}\right)^{*}-N_{q 1 q 2}\right]^{2}$,

and

$$
\begin{aligned}
& C_{1}(I)=-\left[\left(N_{q 2 q 1}\right)^{*}-N_{q 1 q 2}\right]^{2}, \\
& C_{2}(I)=-\left[\left(N_{q 2 q 1}\right)^{*}+N_{q 1 q 2}\right]^{2} .
\end{aligned}
$$



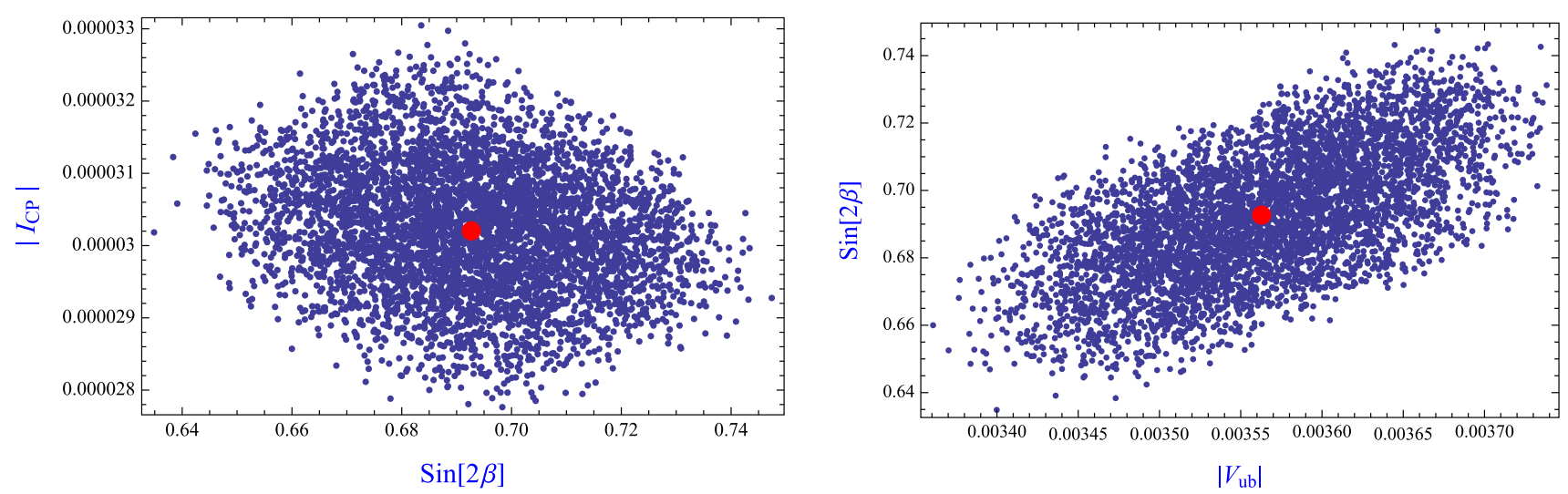

Fig. 1 We present scatter plots showing $\left|I_{\mathrm{CP}}\right|$ versus $\sin 2 \beta$ and $\sin 2 \beta$ versus $\left|V_{u b}\right|$ obtained by varying randomly the input parameters around the reference point

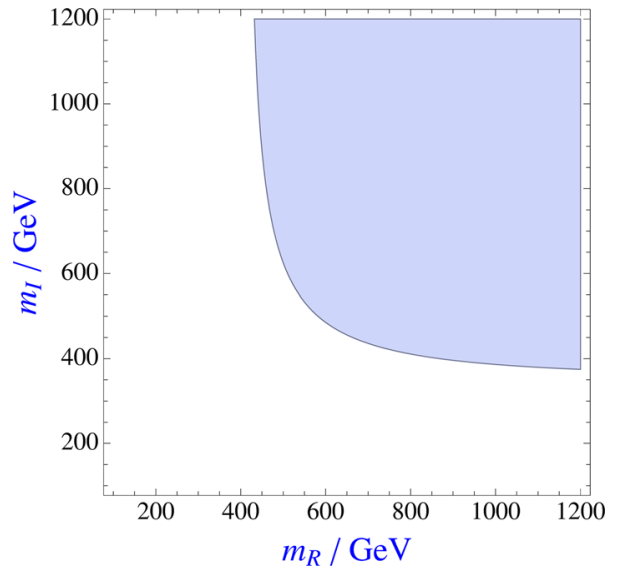

Fig. 2 Plot showing the allowed region for $m_{I}$ and $m_{R}$, taking into account the constraint on $\Delta m_{K}$

The indices $q_{1}$ and $q_{2}$ refer to the valence quarks of the meson $M$, and $N$ is $N_{u}$ or $N_{d}$, depending on the meson system considered.

In this framework it is a good approximation to use the matrix $F$ for both $U_{d_{L}}$ and $U_{d_{R}}$. Using the values we obtained for $\delta_{d}$ and taking, as already discussed, $t \simeq 1$ and $c_{1}^{d} \simeq$ $\frac{\sqrt{2}}{3} \frac{m_{b}}{v}$, the new physics contribution to $M_{12}^{K}$ becomes solely dependent on $f_{K}, m_{K}, m_{R}$ and $m_{I}$. In $K^{0}-\bar{K}^{0}$, both $M_{12}^{K}$ and $\Gamma_{12}^{K}$ are relevant for the mass difference $\Delta m_{K}$. It is reasonable to impose the constraint that $M_{12}^{\mathrm{NP}}$ in the neutral kaon system does not exceed the experimental value of $\Delta m_{K}$. Adopting as input values the PDG experimental determinations of $f_{K}$, $m_{K}$ and $\Delta m_{K}$ [30], one is left with combinations of $m_{R}$ and $m_{I}$ where our model respects the inequality $M_{12}^{N P(K)}<$ $\Delta m_{K}$. The region plot that we have obtained is presented in Fig. 2. It is clear that in this framework the masses of the flavour-violating neutral Higgs can be below the $\mathrm{TeV}$ scale, so that they could be discovered at the next run of the LHC.

\section{Conclusions}

We have studied in detail, in the framework of 2HDM, the question of stability of alignment, under the renormalisation group. It was shown that there are new stable solutions, apart from those found in Ref. [8]. Stability under the RGE puts very strict restrictions on the flavour structure of the Higgs Yukawa couplings. If one imposes the stability conditions and at the same time requires that the flavour structure is in agreement with the observed pattern of quark masses and mixing, then one is lead to a unique solution, where all Higgs flavour matrices are proportional to the so-called democratic matrix. We have also shown that these flavour structures leading to stable alignment can be obtained by imposing on the Lagrangian a $Z_{3} \times Z_{3}^{\prime}$ symmetry. In the limit where this symmetry is exact, only the third generation of quarks acquires a mass. Non-vanishing masses for the two first generations are obtained through the breaking of the discrete symmetry which in turn generates scalar-mediated FCNCs which are suppressed by the smallness of the light quark masses.

It should be pointed out that the proposed framework does not automatically imply a natural suppression of CP violating effects such as edms $[31,32]$. In this respect this framework has the same features as the standard aligned model of Ref. [7].

The scenario presented in this paper provides a natural framework for the alignment hypothesis and establishes a possible intriguing link between stability of alignment in $2 \mathrm{HDM}$ and the observed pattern of quark masses and mixing.

Acknowledgments This work is partially supported by Spanish MINECO under Grant FPA2011-23596, by Generalitat Valenciana under Grant GVPROMETEOII 2014-049 and by Fundação para a Ciência e a Tecnologia (FCT, Portugal) through the Projects CERN/FP/123580/2011, PTDC/FIS-NUC/0548/2012, EXPL/FIS-NUC /0460/2013 and CFTP-FCT Unit 777 (PEst-OE/FIS/UI0777/2013), which are partially funded through POCTI (FEDER), COMPETE, QREN and EU. 
Open Access This article is distributed under the terms of the Creative Commons Attribution 4.0 International License (http://creativecomm ons.org/licenses/by/4.0/), which permits unrestricted use, distribution, and reproduction in any medium, provided you give appropriate credit to the original author(s) and the source, provide a link to the Creative Commons license, and indicate if changes were made.

Funded by SCOAP ${ }^{3}$.

\section{Appendix: Solutions to the alignment conditions}

The solutions to the alignment conditions

$\Omega_{1} \Omega_{1}^{\dagger} \Gamma_{1}=\lambda_{\Gamma} \Gamma_{1} ; \quad \Gamma_{1} \Gamma_{1}^{\dagger} \Omega_{1}=\lambda_{\Omega} \Omega_{1}$

can be obtained by the following steps. First we define the Hermitian matrices

$H_{\Gamma}=\Gamma_{1} \Gamma_{1}^{\dagger} ; \quad H_{\Omega}=\Omega_{1} \Omega_{1}^{\dagger}$.

It is easy to show that $\lambda_{\Gamma}$ and $\lambda_{\Omega}$ are real. This can be achieved by multiplying the first equation by its Hermitian conjugate and inserting the second equation (and vice versa) to get

$\lambda_{\Omega} H_{\Omega}^{2}=\left|\lambda_{\Gamma}\right|^{2} H_{\Gamma}$,

$\lambda_{\Gamma} H_{\Gamma}^{2}=\left|\lambda_{\Omega}\right|^{2} H_{\Omega}$;

it follows from these equations that both $\lambda_{\Omega}$ and $\lambda_{\Gamma}$ should be real since one has two identities among Hermitian matrices. Now multiplying each of the Eq. (42) on the right by $\Gamma_{1}^{\dagger}$ and $\Omega_{1}^{\dagger}$, respectively, we get

$H_{\Omega} H_{\Gamma}=\lambda_{\Gamma} H_{\Gamma} ; \quad H_{\Gamma} H_{\Omega}=\lambda_{\Omega} H_{\Omega}$,

and taking Hermitian conjugates

$H_{\Gamma} H_{\Omega}=\lambda_{\Gamma} H_{\Gamma} ; \quad H_{\Omega} H_{\Gamma}=\lambda_{\Omega} H_{\Omega} ;$

therefore,

$\lambda_{\Gamma} H_{\Gamma}=\lambda_{\Omega} H_{\Omega}$,

and we conclude that

$\left[H_{\Gamma}, H_{\Omega}\right]=0$,

implying that $V_{\mathrm{CKM}}=I$ up to permutations of rows or columns. Denoting the usual bi-unitary diagonalisation procedure by

$\Gamma_{1}=V_{L}^{\Gamma} D_{\Gamma} V_{R}^{\Gamma \dagger} ; \quad \Omega_{1}=V_{L}^{\Omega} D_{\Omega} V_{R}^{\Omega \dagger}$,

from Eq. (49) we conclude that we can always choose the unitary matrices $V_{L}^{\Gamma}$ and $V_{L}^{\Omega}$ equal to each other,

$V_{L}^{\Gamma}=V_{L}^{\Omega}$ and the alignment conditions can easily be reduced to conditions among the diagonal matrices $D_{\Gamma}$ and $D_{\Omega}$. From Eq. (46), it then follows that

$D_{\Omega}^{2} D_{\Gamma}=\lambda_{\Gamma} D_{\Gamma} ; \quad D_{\Gamma}^{2} D_{\Omega}=\lambda_{\Omega} D_{\Omega}$.

It can be checked that there are only two types of solutions. Those with $\lambda_{\Gamma}$ and $\lambda_{\Omega}$ different from zero (solutions 1-3) and the remaining ones (solutions 4,5 ).

1. $D_{\Gamma}=a P_{3}$ and $D_{\Omega}=\alpha P_{3}$ and changes of $P_{3}$ by $P_{2}$ or $P_{1}$.

2. $D_{\Gamma}=a\left(I-P_{1}\right)$ and $D_{\Omega}=\alpha\left(I-P_{1}\right)$ and changes of $P_{1}$ by $P_{2}$ or $P_{3}$.

3. $D_{\Gamma}=a I$ and $D_{\Omega}=\alpha I$.

4. $D_{\Gamma}=0$ and $D_{\Omega}$ arbitrary and vice versa.

5. $D_{\Gamma}=a P_{i}$ and $D_{\Omega}=\alpha\left(I-P_{i}\right)$

where $P_{i}$ stand for the projection operators

$\left(P_{i}\right)_{j k}=\delta_{i j} \delta_{i k}$.

Solutions 2-4 cannot be good approximations to the actual quark spectra due to the implied degeneracy. Solution 5 gives rise to $V_{\mathrm{CKM}}$ matrix very different from the identity matrix. Only solution 1 provides, in leading approximation the correct pattern of quark masses and mixing. In a suitable weak basis, this solution can be written as a democratic matrix $\Delta$.

\section{References}

1. T.D. Lee, A theory of spontaneous T violation. Phys. Rev. D 8, $1226(1973)$

2. G.C. Branco, P.M. Ferreira, L. Lavoura, M.N. Rebelo, M. Sher, J.P. Silva, Theory and phenomenology of two-Higgs-doublet models. Phys. Rep. 516, 1 (2012). arXiv:1106.0034 [hep-ph]

3. A. Crivellin, A. Kokulu, C. Greub, Flavor-phenomenology of twoHiggs-doublet models with generic Yukawa structure. Phys. Rev. D 87(9), 094031 (2013). arXiv:1303.5877 [hep-ph]

4. S.L. Glashow, S. Weinberg, Natural conservation laws for neutral currents. Phys. Rev. D 15, 1958 (1977)

5. G.C. Branco, Spontaneous CP nonconservation and natural flavor conservation: a minimal model. Phys. Rev. D 22, 2901 (1980)

6. G.C. Branco, M.N. Rebelo, The Higgs mass in a model with two scalar doublets and spontaneous CP violation. Phys. Lett. B 160, 117 (1985)

7. A. Pich, P. Tuzon, Yukawa alignment in the two-Higgs-doublet model. Phys. Rev. D 80, 091702 (2009). arXiv:0908.1554 [hep$\mathrm{ph}]$

8. P.M. Ferreira, L. Lavoura, J.P. Silva, Renormalization-group constraints on Yukawa alignment in multi-Higgs-doublet models. Phys. Lett. B 688, 341 (2010). arXiv:1001.2561 [hep-ph]

9. H. Serodio, Yukawa alignment in a multi Higgs doublet model: an effective approach. Phys. Lett. B 700, 133 (2011). arXiv:1104.2545 [hep-ph] 
10. I. de Medeiros, Varzielas, Family symmetries and alignment in multi-Higgs doublet models. Phys. Lett. B 701, 597 (2011). arXiv:1104.2601 [hep-ph]

11. A. Celis, J. Fuentes-Martin, H. Serodio, Effective aligned 2HDM with a DFSZ-like invisible axion. Phys. Lett. B 737, 185 (2014). arXiv:1407.0971 [hep-ph]

12. G.C. Branco, W. Grimus, L. Lavoura, Relating the scalar flavor changing neutral couplings to the CKM matrix. Phys. Lett. B 380, 119 (1996). arXiv:hep-ph/9601383

13. F.J. Botella, G.C. Branco, M. Nebot, M.N. Rebelo, Two-Higgs leptonic minimal flavour violation. JHEP 1110, 037 (2011). arXiv:1102.0520 [hep-ph]

14. F.J. Botella, G.C. Branco, M.N. Rebelo, Minimal flavour violation and multi-Higgs models. Phys. Lett. B 687, 194 (2010). arXiv:0911.1753 [hep-ph]

15. G. Bhattacharyya, D. Das, P.B. Pal, M.N. Rebelo, Scalar sector properties of two-Higgs-doublet models with a global $U(1)$ symmetry. JHEP 1310, 081 (2013). arXiv:1308.4297 [hep-ph]

16. F.J. Botella, G.C. Branco, A. Carmona, M. Nebot, L. Pedro, M.N. Rebelo, Physical constraints on a class of two-Higgs doublet models with FCNC at tree level. arXiv:1401.6147 [hep-ph]

17. G. Bhattacharyya, D. Das, A. Kundu, Feasibility of light scalars in a class of two-Higgs-doublet models and their decay signatures. Phys. Rev. D 89, 095029 (2014). arXiv:1402.0364 [hep-ph]

18. T.P. Cheng, M. Sher, Mass matrix ansatz and flavor nonconservation in models with multiple Higgs doublets. Phys. Rev. D 35, 3484 (1987)

19. G.C. Branco, J.I. Silva-Marcos, M.N. Rebelo, Universal strength for Yukawa couplings. Phys. Lett. B 237, 446 (1990)

20. H. Fritzsch, Lepton - quark masses and democratic symmetry. Nucl. Phys. Proc. Suppl. 40, 121 (1995). arXiv:hep-ph/9411419

21. G.C. Branco, J.I. Silva-Marcos, Predicting V (CKM) with universal strength of Yukawa couplings. Phys. Lett. B 359, 166 (1995). arXiv:hep-ph/9507299

22. W. Grimus, L. Lavoura, Renormalization of the neutrino mass operators in the multi-Higgs-doublet standard model. Eur. Phys. J. C 39, 219 (2005). arXiv:hep-ph/0409231
23. C.B. Braeuninger, A. Ibarra, C. Simonetto, Radiatively induced flavour violation in the general two-Higgs doublet model with Yukawa alignment. Phys. Lett. B 692, 189 (2010). arXiv:1005.5706 [hep-ph]

24. M. Jung, A. Pich, P. Tuzon, Charged-Higgs phenomenology in the aligned two-Higgs-doublet model. JHEP 1011, 003 (2010). arXiv: 1006.0470 [hep-ph]

25. G. Cvetic, C.S. Kim, S.S. Hwang, Higgs mediated flavor changing neutral currents in the general framework with two Higgs doublets: an RGE analysis. Phys. Rev. D 58, 116003 (1998). arXiv:hep-ph/9806282

26. A.J. Buras, M.V. Carlucci, S. Gori, G. Isidori, Higgs-mediated FCNCs: natural flavour conservation vs. minimal flavour violation. JHEP 1010, 009 (2010). arXiv: 1005.5310 [hep-ph]

27. G.C. Branco, J.I. Silva-Marcos, Invariants, alignment and the pattern of fermion masses and mixing. Phys. Lett. B 715, 315 (2012). arXiv:1112.1631 [hep-ph]

28. S. Antusch, V. Maurer, Running quark and lepton parameters at various scales. JHEP 1311, 115 (2013). arXiv:1306.6879 [hep-ph]

29. J. Charles, A. Hocker, H. Lacker, S. Laplace, F.R. Le Diberder, J. Malcles, J. Ocariz, M. Pivk, L. Roos (CKMfitter Group Collaboration), $\mathrm{CP}$ violation and the $\mathrm{CKM}$ matrix: assessing the impact of the asymmetric B factories. Eur. Phys. J. C 41, 1-131 (2005). doi:10.1140/epjc/s2005-02169-1. arXiv:hep-ph/0406184

30. K.A. Olive et al. (PDG Collaboration), Review of particle physics. Chin. Phys. C 38, 090001 (2014)

31. M. Raidal, A. van der Schaaf, I. Bigi, M.L. Mangano, Y.K. Semertzidis, S. Abel, S. Albino, S. Antusch et al., Flavour physics of leptons and dipole moments. Eur. Phys. J. C 57, 13 (2008). arXiv:0801.1826 [hep-ph]

32. M. Jung, A. Pich, Electric dipole moments in two-Higgs-doublet models. JHEP 1404, 076 (2014). arXiv:1308.6283 [hep-ph] 